\title{
REALIZATION AND CHARACTERIZATION OF A FOUR-CHANNEL INTEGRATED OPTICAL YOUNG INTERFEROMETER
}

\author{
Aurel Ymeti ${ }^{a}$, Johannes S. Kanger ${ }^{a}$, Jan Greve ${ }^{a}$, Paul V. Lambeck ${ }^{b}$, Robert Wijn ${ }^{c}$, and Rene G. Heideman ${ }^{c}$ \\ Biophysical Tcchniques ${ }^{a}$, Lightwave Device Group ${ }^{\mathrm{b}}, \mathrm{MES}^{+}{ }^{+}$Institute, University of Twente, P.O. Box 217, \\ 7500 AE Enschede, The Netherlands; Lionix b.v. ${ }^{c}$, P.O. Box 456, 7500 AE Enschede, The Netherlands \\ Tel.: +3153 4893112,Fax: +31534891105, e-mail: A.Ymeti@utwente.nl
}

\begin{abstract}
In this paper, we report the realization and characterization of a four-channel integrated optical Young interferometer (YI), which enables simultaneous and independent monitoring of three binding processes. The simultaneous and independent measurement of three different glucose concentrations shows the multi-purpose feature of such device. The phase resolution for different pairs of channels was $\sim 1 \times 10^{-4}$ fringes, which corresponds to a refractive index resolution of $-8.5 \times 10^{-8}$. The observed errors, which are caused due to mismatching of spatial frequencies of individual interference patterns with those determined from the $\mathrm{CCD}$ camera, have been reduced by using different reduction schemes. In addition, we have investigated a novel method for discrimination of the refractive index change from the thickness of a bound layer during an immunoreaction, as well as measuring the temperature change that takes place during such a process.
\end{abstract}

\section{INTRODUCTION}

A common feature of the interference-based sensors that have been developed in recent years, such as MZI [1], YI [2], etc, is that they are able to monitor one single analyte at a time, e.g. concentration of a given pesticide in drinking water [3]. It is desirable to expand these sensitive sensors to a multichannel device, which could enable monitoring of multiple binding processes simultaneously and independently from each other by immobilizing different receptor molecules to different channels. In addition, by using such a device, a binding curve of an analyte to a specific receptor layer can be achieved by applying different concentrations of the analyte molecules to different channels. Several measurement devices can be used simultaneously to fulfill this objective, but a more attractive option is the use of a sensor that contains several sensing elements in parallel. Advantages of such device can be simple addressing of all sensors using one light source, easier sample handling, less packaging costs, etc.

\section{THEORETICAL DESCRIPTION}

Four-channel YI. The principle of the 4-ch YI is similar to that of the 2-ch YI as described previously [4]. In our integrated 4-ch YI, monochromatic laser light with a vacuum wavelength $\lambda$ is coupled into an input channel waveguide and equally split to 4 output parallel channels, as shown in Figure 1.

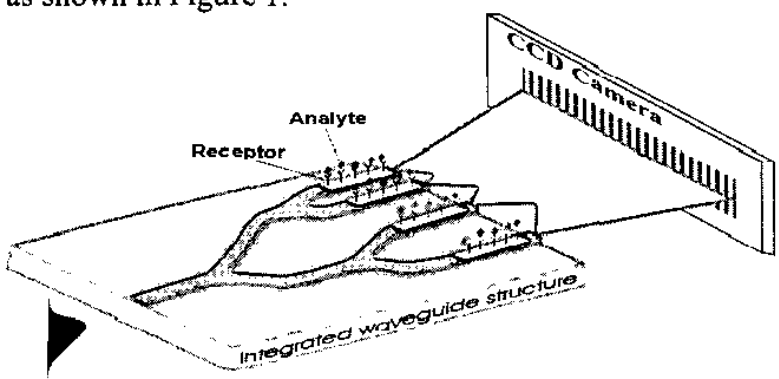

Figure 1. An artistic impression of the 4-ch integrated optical YI.

The output divergent beams will overlap with each other and the final interference pattern will be a superposition of individual interference patterns, each of them representing the overlap of the divergent beams of a specific channel pair. The interference pattern is recorded by a CCD camera, which is placed at a distance $L$ from endface of the integrated chip. The irradiance distribution of the sum interference pattern at the input face of the camera can be derived to be

$$
\mathrm{I}(\mathrm{y})=4 \mathrm{I}_{0}+2 \mathrm{I}_{0} \sum_{\mathrm{i}, j=\mathrm{i} ; i<j}^{4} \cos \left(\Delta \Phi_{\mathrm{ij}}(y)+\Delta \varphi_{\mathrm{ij}}\right)
$$

provided output powers of four channel outputs are identical. A channel output is approximated as a point source. In Eq. (1), $\Delta \Phi_{i j}(y)$ is the phase difference between the interfering beams coupled out from $i$ th and $j$ th channels as a result of their different optical path length at the interfering position $y$ on the CCD camera; $d_{i j}$ is the distance between these channels and $\Delta \varphi_{i j}$ is the phase change induced by refractive index changes $\Delta n_{i}$ and $\Delta n_{j}$ in $i$ th and $j$ th channels respectively. The phase change $\Delta \Phi_{i j}(\mathrm{y})$ can be derived to be [5]

$$
\Delta \Phi_{i j}(y)=\frac{2 \pi}{\lambda} \frac{d_{i j}}{L} y
$$

provided that $d_{i j} \ll<$ for all $i, j$ values, a condition that is well satisfied in our case. Hence, an individual 
interference pattern shows a spatial frequency $k_{i j}=d_{i j} /(\lambda L)$. The refractive index changes in channels $i$ and $j$ can be induced due to a binding process between analyte and receptor molecules within the evanescent region of a guided mode in the sensing window of each channel. The phase change $\Delta \varphi_{i j}$ can be written as

$$
\Delta \varphi_{i j}=\frac{2 \pi}{\lambda} l \frac{\partial N_{e f f}}{\partial n} \Delta n_{i j}
$$

where $l$ is the length of the sensing window realized on top of each output channel, $\partial N_{\text {eff }} \partial n$ is the sensitivity of the effective refractive index $N_{\text {eff }}$ to the refractive index change, and $\Delta n_{i j} \equiv \Delta n_{i}-\Delta n_{j}$. A change of $\Delta n_{i j}$ causes a spatial shift $\Delta y_{i j}$ of the individual interference pattern for this channel pair along the camera surface. This shift can be calculated in similar way as for the 2-ch YI as

$$
\Delta n_{i j}=\frac{d_{i j}}{l L}\left(\frac{\partial N_{e f f}}{\partial n}\right)^{-1} \Delta y_{i j}
$$

If one chooses channel one, two, and three to be the measuring channels, and channel four as the reference channel, the refractive index changes $\Delta n_{14}, \Delta n_{24}$, and $\Delta n_{34}$ can be determined from Eq. (4) by measuring the spatial shifts $\Delta y_{14}, \Delta y_{24}$, and $\Delta y_{34}$. These spatial shifts can be measured simultaneously and independently from each other by choosing different distances $d_{i j}$ between output parallel channels such that each pair of channels can be considered as a 2-ch YI with a unique distance between its measuring and reference arms [4].

Analysis of phase error and cross-talk. The simulations with the 4-ch YI have shown a difference between the expected phase changes and resulting ones if a given phase change is introduced at channel 1 . This difference is noted as phase error (PE). In addition, a phase change is observed for the channel pairs where no phase change is expected, noted as cross-talk (CT). The origin of these errors can be found in the signal analysis algorithm, a problem that is well-recognized in literature $[6,7]$.

In the 4-ch YI the interference pattern is recorded with a CCD camera, which has a finite number of pixels that are equally spaced from each other, and analyzed based on a FFT algorithm. According to the Discrete Fourier Transform (DFT) theory [8], the outcome of the Fourier transformation will be a discrete spectrum of spatial frequencies, which are determined by the total number of camera pixels and the sampling interval. On the other hand, the spatial frequency of the interference pattern between two output channels $i$ and $j$ depends on their distance $d_{\mathrm{ij}}$, the wavelength used, and the distance between the CCD camera and chip endface. For a particular set of these parameter values, the spatial frequency of a given interference pattern can be either equal to one of the spatial frequencies determined from the CCD camera, or different from them. The distance of the CCD camera from the chip endface where all spatial frequencies of individual interference patterns match with one of the spatial frequencies determined from the $\mathrm{CCD}$ camera is noted as a 'matching' distance. It is known that if the frequency of the original signal matches a spatial frequency of the CCD camera exactly, only the main lobe of the spectrum is present. In that case, the frequency of the original signal can be read very precisely, and so can the phase of the signal. On the other hand, if this condition is not satisfied [9], the energy from the main peak leaks out to the surrounding frequencies, known as spectral leakage, resulting in the appearance of side-lobes in the spectrum. For signals containing more than one spatial frequency, side-lobes of one frequency can overlap with main lobes of other frequencies, introducing in such a way an additional error in the positioning of the main lobe. In the 4-ch YI, where the original signal contains six individual interference patterns, resulting in six different spatial frequencies, this effect is significant. Consequently, an error is introduced on extracting the corresponding phase values at the phase part of the FFT, giving rise to the $\mathrm{PE}$ and $\mathrm{CT}$.

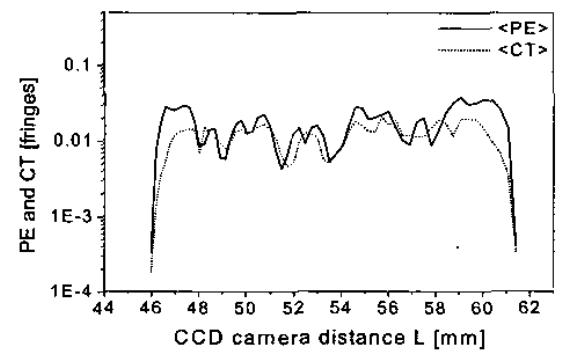

Figure 2. Calculated average $\mathrm{PE},\langle\mathrm{PE}\rangle$, and $\mathrm{CT},\langle\mathrm{CT}\rangle$, in the 4-ch YI, when a phase change of $0.5 \times 2 \pi$ is introduced in channel 1 . See text for further details.

In Figure 2 is calculated the PE and CT when a phase change of $0.5 \times 2 \pi$ is introduced in channel 1 of the 4-ch YI. The calculations show that the resulting PE and CT are reduced to $\sim 0$ at the 'matching' distances of 45.9 and $61.4 \mathrm{~mm}$, while they can grow up to values of $0.04 \times 2 \pi$ at intermediate distances. This result clearly shows that the PE and CT are minimized when a match between the spatial frequencies of the individual interference patterns in the 4-ch YI and the spatial frequencies determined by the $\mathrm{CCD}$ camera, is implemented.

In Figure 3 are shown the PE and CT calculated for different phase changes introduced in channel 1 and no spatial frequency matching is present. According to this calculation, the PE and CT are reduced to $\sim 0$ when the introduced phase change at the output channels is an integer number of fringes, regardless of the spatial frequency matching condition. Moreover, the $\mathrm{PE}$ and $\mathrm{CT}$ are periodically changing with a period of $2 \pi$ and they reach a maximum when the introduced phase change at 
output channel is around $(N+0.5) \times 2 \pi$, where $N$ is an integer number.

Different techniques can be used to reduce the PE and CT in the 4-ch YI, such as adjustment of the setup geometry with the purpose of achieving a spatial frequency matching, e.g. by adjusting the CCD camera distance; application of windowing technique [10] where the recorded interference pattern is multiplied with a window function, e.g. the Hanning window, before the FFT is applied; implementation of the spatial frequency matching in the algorithm, which consists of truncating the recorded interference pattern such that an integer number of fringes is contained for each individual interference pattern. For that purpose, it is often necessary to resample initially the signal data to a larger number of data points using an interpolation step, such that after the truncation procedure, the total number of fringes will be comprised by an integer number of data points.

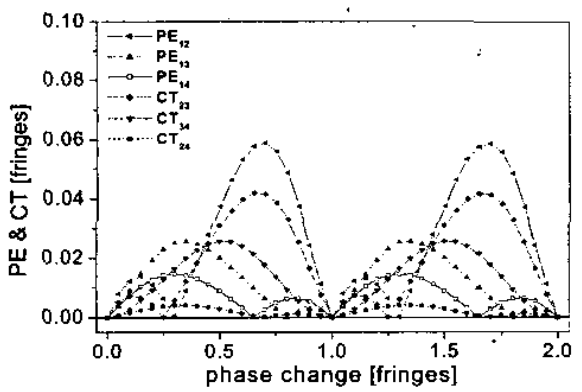

Figure 3. Calculated PE $\left(\mathrm{PE}_{1 \mathrm{i}}, \mathrm{i}=2,3,4\right)$ and $\mathrm{CT}\left(\mathrm{CT}_{\mathrm{ij}}, \mathrm{i}, \mathrm{j}\right.$ $\neq 1$ ) in the 4-ch YI if the phase change introduced in channel 1 is gradually increased from 0 to $2 \times 2 \pi$.

According to our calculations, when the Hanning window is used, the PE and CT can be reduced with a factor of $~$ 5. Using the matching technique, the PE and CT in the four-channel YI can also be reduced with a factor of $\sim 5$.

\section{CHIP FABRICATION AND SET-UP}

The 4-ch integrated optical YI chip has been realized as an optical circuit consisting of three $\mathrm{Y}$ junctions positioned in such a way that the two output channels of the first $\mathrm{Y}$-junction serve as input channels for the next two ones, as shown in Figure 4. The distance between the output branches of the first Y-junction was chosen $160 \mu \mathrm{m}$ such that the distances between the four output channels are $d_{12}=60, d_{23}=80$ and $d_{34}=100 \mu \mathrm{m}$ guaranteeing that all six spatial frequencies in the interference pattern are different. The $\mathrm{Y}$-junctions have been designed with $\mathrm{S}$-bend structures having a bend radius of $10 \mathrm{~mm}$. The bend in the input waveguide is implemented for reducing the intensity of the slab light at the channel outputs. The channel waveguide structure has been realized in SiON technology [11]. The layer structure consists of a silicon $<100\rangle$ wafer provided with a $3 \mu \mathrm{m}$ thick thermal silicon oxide layer with a refractive index $n_{s}=1.459$, a $90 \mathrm{~nm}$ thick LPCVD grown silicon nitride core layer with refractive index of $n_{F}=2.036$, and a $1.5 \mu \mathrm{m}$ deposited PECVD silicon oxide layer with refractive index $n_{C}=1.464$. All refractive indices refer to a vacuum wavelength of $647 \mathrm{~nm}$. The ridge of the channel waveguide structure and the channel width have been chosen respectively $1 \mathrm{~nm}$ and $2 \mu \mathrm{m}$, guaranteeing a single-mode behavior for the TE polarized light with a vacuum wavelength of $647 \mathrm{~nm}$. A sensing window is realized on top of each output channel, as shown in Figure 4 , by locally removing the PECVD $\mathrm{SiO}_{2}$ layer using a BHF etching solution. Each sensing window is 4 $\mathrm{mm}$ long and $100 \mu \mathrm{m}$ wide. Sensing windows are positioned in a 'diagonal' configuration with an interdistance of $3 \mathrm{~mm}$.

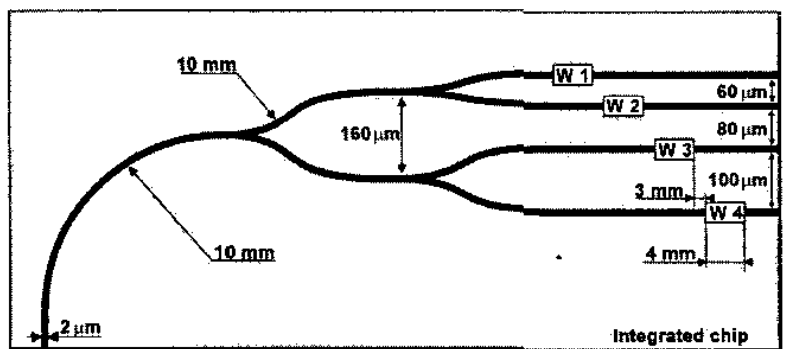

Figure 4. Top-view of the 4-ch integrated YI chip; W 1, $\mathrm{W} 2, \mathrm{~W} 3$, and $\mathrm{W} 4$, are the sensing window on channel 1 , 2,3 and 4 respectively.

Monochromatic laser light with a wavelength of $647 \mathrm{~nm}$ is end-fire coupled into the input channel-waveguide using a microscope objective with numerical aperture of 0.65 . The four output divergent beams are overlapping each other at the front side of a CCD camera (Teli CS$3440 ; 756 \times 575$ pixels; $w_{\text {pixel }}=11.6 \mu \mathrm{m}$ ), which is placed at a distance of about $60 \mathrm{~mm}$ from the chip endface and parallel to the endface plane for recording the interference pattern. A cylindrical lens is positioned between the chip endface and the CCD camera to collimate the output divergent beams in the direction perpendicular to the sensor chip, increasing in that way the light intensity that reaches the camera. A four-chamber flow-through cuvette is gently pressed on the chip such that sampling liquids can independently flow into each chamber. The recorded sum interference pattern is digitized to 12-bit information, and analyzed by a computer program in which a 2D-FFT algorithm is implemented. This algorithm consists of selecting six different peaks, which correspond to six pair of channels, in the amplitude of the Fast Fouriertransformed interference pattern. At the phase part of the FFT the phase value corresponding to each pair of channels is extracted at the given spatial frequencies.

\section{MEASUREMENTS}

In Figure 5 is shown the response of the sensor when the phase change introduced in channel 1 is gradually increased from 0 to $2 \times 2 \pi$ by flowing $1.232 \%$ (by weight) glucose solution [12]. The PE and CT measured for 
different phase changes introduced in channel 1 are shown in Figure 6. These errors might be caused by experimental errors such as drift, misalignment, or leakage of the four flow chambers, but also by the errors introduced by the phase extraction algorithm, as we concluded from calculations.

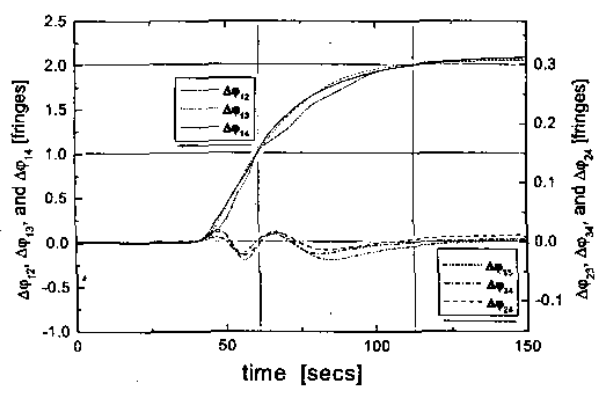

Figure 5. Time-response of the 4-ch YI sensor to a phase change of $2 \times 2 \pi$ introduced in channel 1 by flowing $1.232 \%$ (by weight) glucose solution.

At phase changes of an integer number of fringes the PE and $\mathrm{CT}$ are caused only by experimental errors. We observe that $\mathrm{PE}$ and $\mathrm{CT}$ are nearly zero $(-0.005 \times 2 \pi)$ for phase changes of $2 \pi$ and $2 \times 2 \pi$. This is a clear indication that experimental errors are small, and the deviations observed in Figure 6, are largely due to the data analysis algorithm.

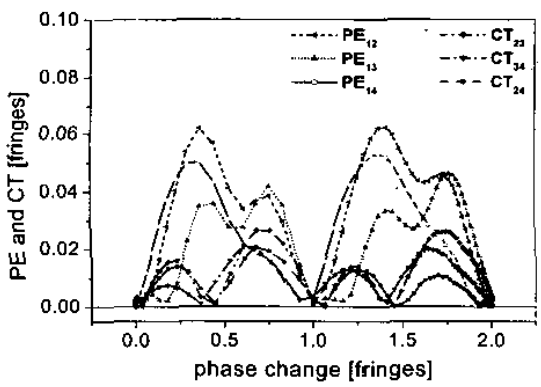

Figure 6. Measured $P E\left(\mathrm{PE}_{1 \mathrm{i}} \mathrm{i}=2,3,4\right)$ and $\mathrm{CT}\left(\mathrm{CT}_{\mathrm{ij}} \mathrm{i}, \mathrm{j} \neq 1\right)$ vs. the phase change introduced in channel 1 (see Fig. 5).

In order to reduce the PE and CT for phase changes different from integer number of fringes occurring at output channels of the device, several techniques described above are implemented. If the windowing technique is applied, using Hamming window the PE and CT are respectively reduced with a factor of $~$ 4 and $~ 1.4$. The PE and CT are reduced with a factor of $~$ 1.9 and $\sim 2.4$ respectively if the technique that aims at the removal of the mismatching effect is implemented. A better result is achieved by combining two last techniques, which can decrease both the PE and CT with a factor of $\sim 3.5$. The experimentally obtained PE and CT reductions are smaller than indicated by the calculations. The reason can be found in the presence of spurious fringes in the real interference pattern and a nonlinear response of the detector $[6,7]$, which are not assumed in the calculations. In addition, one has to consider the influence of experimental errors such as drift, misalignment, or any leakage of the flow chambers, which again are not included in calculations.

In Figure 7 the response of the sensor is shown when a phase change of $2 \pi$ is introduced in channel one and three, and a phase change of $2 \times 2 \pi$ is introduced in channel two simultaneously, using $0.616 \%$ and $1.232 \%$ (by weight) glucose solutions. Pure water is continuously flown to channel four. After a stable signal has been reached, the solutions were exchanged for pure water, at first in channel two, next in channels three and one.

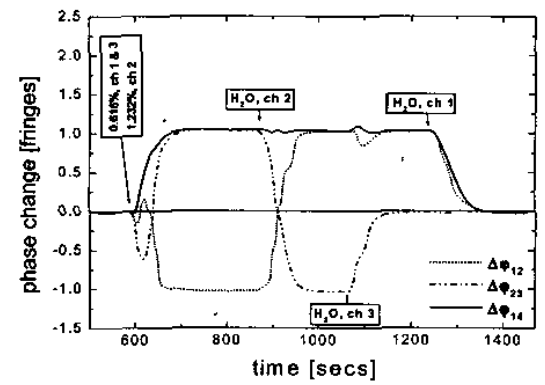

Figure 7. Phase changes $\Delta \varphi_{12}, \Delta \varphi_{23}$, and $\Delta \varphi_{14}$ measured as a function of time in the 4-ch YI when a phase change of $2 \pi$ is introduced in channel 1 and 3 and simultaneously a phase change of $2 \times 2 \pi$ in channel 2 .

From the measured values, $\Delta \varphi_{12}, \Delta \varphi_{23}$, and $\Delta \varphi_{14}$, we can determine the phase change that is introduced in the channels, $\Delta \phi_{1}, \Delta \phi_{2}$, and $\Delta \phi_{3}$. Note that $\Delta \phi_{4}=0$, because no phase change is introduced in channel four. The timedependence of these channel phase changes is shown in Figure 8 . The obtained phase changes nicely correspond to the introduced ones, in that way demonstrating one of the multi-purpose features of the multichannel device with which, three different glucose concentrations were measured simultaneously and independently from each other.

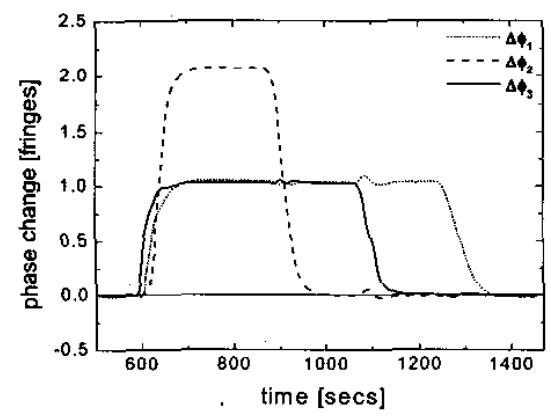

Figure 8. Phase changes $\Delta \phi_{1}, \Delta \phi_{2}$, and $\Delta \phi_{3}$ calculated from the measured phase changes shown in Figure 7.

The phase resolution, as determined from these measurements, is $\sim 1 \times 10^{-4}$ fringes, corresponding to an effective refractive index resolution of $\sim 1.6 \times 10^{-8}$ (refractive index change of $-8.5 \times 10^{-8}$ ). The measured 
long-term stability of the phase change for different pair of channels is $-5 \times 10^{-4}$ fringes $\times \mathrm{h}^{-1}$, being improved compared to the one in the 2-ch YI $\left(\sim 1 \times 10^{-3}\right.$ fringes $\left.\mathrm{x} \mathrm{h}^{-1}\right)$ because the environmental changes, such as temperature fluctuations, are better compensated due to the presence of a sensing window on top of each output channel.

\section{MULTIPLE.WAVELENGTH APPROACH}

The interference-based sensor, such as MZI, YI, etc. are used to measure the phase change between the measuring and the reference channels when a bulk refractive index change, or a thickness change of a bound layer in the sensing window of the measuring channel occur, see Figure 9. It is often possible that these two contributions occur simultaneously and the resulting phase change is a sum of both contributions. In addition, the influence of factors such as temperature changes, which can be caused during an immunoreaction, introduce an extra phase change which is added to the one caused from the binding process. In order to reduce such an influence, the reference and measuring channels are usually positioned close to each other in order to compensate the possible temperature changes. However, this influence cannot be completely avoided, especially at. short time intervals. after the immunosensing process occurs. It will be a more attractive alternative if one can determine the phase change due to the temperature changes, which later can be subtracted from the phase change caused by the binding event.

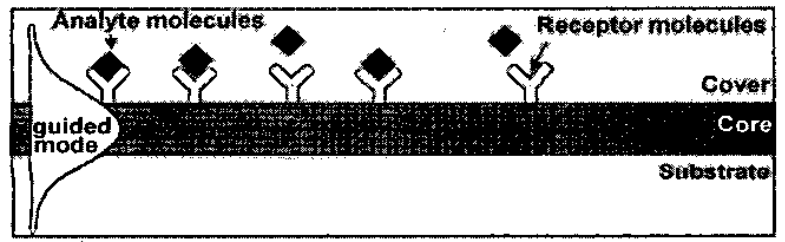

Figure 9. Schematic presentation of a three-layer waveguide structure where a binding process between analyte and receptor molecules is taking place at the corecover interface.

We have found, for the first time to our knowledge, a method to determine simultaneously and separately the phase changes due to the bulk refractive index change and the thickness change of a bound layer, including the one due to the temperature change. This approach consists of simultaneous use of three different wavelengths, and because of the dispersion effect, three different phase changes can simultaneously and independently be measured. Consequently, a system of three equations can be written based on which three unknowns, i.e. the refractive index change, thickness change of the bound layer, and temperature change, can be determined. To be able to solve this system of equations, the dependence of the effective refractive index change of a guided mode from a temperature change during an immunoreaction and concentration of the cover medium are derived.

\section{CONCLUSIONS}

A 4-ch integrated optical YI sensor has been designed, fabricated, and tested as the first multichannel interferometer device. We have shown experimentally that the 4-ch YI can be used to measure three different concentrations of an analyte, such as glucose, simultaneously and independently from each other. The measured phase resolution that corresponds to different pair of channels is $\sim 1 \times 10^{-4}$ fringes, corresponding to an effective refractive index resolution of $-1.6 \times 10^{-8}$. Different error reduction schemes, such as the Hamming window and the matching technique, can reduce the PE and CT due to mismatch between the spatial frequencies of the individual interference patterns and those determined from the CCD camera with a factor of $\sim 3.5$.

Furthermore, we have investigated a method to discriminate the refractive index change from the thickness of a bound layer, taking place in an immunoreaction, as well as to measure the temperature change during such a process.

\section{References}

[1] R.G. Heideman, and P.V. Lambeck, "Remote optochemical sensing with extreme sensitivity: design, fabrication and performance of a pigtailed integrated optical phase-modulated Mach-Zehnder interferometer system," Sensors and Actuators B, 61, 100-127 (1999).

[2] A. Brandenburg, "Differential refractometry by an integrated-optical Young interferometer," Sensors and Actuators B, 38-39, 266-271 (1997).

[3] E.F. Schipper, R.P.H. Kooyman, R.G. Heideman, and J. Greve, "Feasibility of optical waveguide immunosensors for pesticide detection: physical aspects," Sensors and Actuators B, 90, 24-25 (1995).

[4] A.Ymeti, J.S. Kanger, R.Wijn, P.V. Lambeck, and J. Greve, "Development of a multichannel integrated interferometer immunosensor," Sensors and Actuators B, 83, 1-7 (2002).

[5] E. Hecht, Optics, Addison Wesley, New York, 1998.

[6] S. Nakadate, "Phase detection of equidistant fringes for highly sensitive optical sensing. I. Principle and error analysis," J. Opt. Soc. Am. A, 5, 1258-1264 (1988).

[7] M. Kujawinska, and J. Wojciak, "High accuracy Fourier Transform fringe pattern analysis," Optics and Lasers in Engineering, 14, 325-339 (1991).

[8] R.N. Bracewell, The Fourier Transform and its Applications, McGraw-Hill, New York, 1965.

[9] M. Cerna, A.F. Harvey, "The fundamentals of FFT-based signal analysis and measurement," Application Note 041, National Instruments, (2000).

[10] F.J. Harris, "On the use of windows for harmonic analysis with the discrete Fourier transform," in Proceedings of IEEE 66, New York, 1978, pp. 51-83.

[11] K. Wörhoff, A. Driessen, P.V. Lambeck, L.T.H. Hilderink, P.W.C. Linders, and T.J.A. Popma, "Plasma enhanced chemical vapour deposition of silicon oxynitride optimized "for application in integrated optics," Sensors and Actuators B, 74, 9-12 (1999).

[12] R.C. Weast, Handbook of Chemistry and Physics, 65th edition, CRC Press, Boca Raton, 1984-1985. 